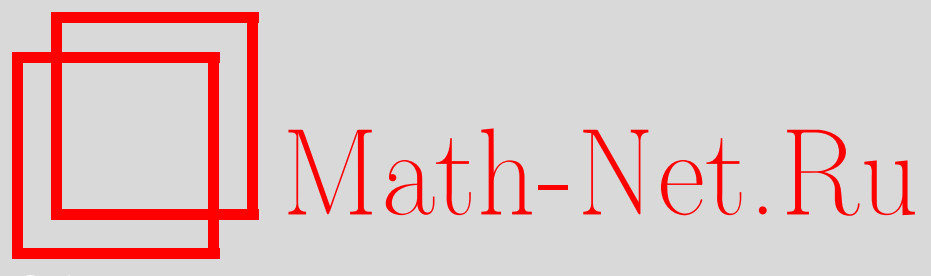

С. П. Суетин, Сильная асимптотика нулей многочленов, ортогональных относительно комплексного веса, УМH, 2007, том 62, выпуск 4, 177-178

DOI: https://doi.org/10.4213/rm7151

Использование Общероссийского математического портала Math-Net.Ru подразумевает, что вы прочитали и согласны с пользовательским соглашением http://www . mathnet.ru/rus/agreement

Параметры загрузки:

IP: 52.23 .180 .231

26 апреля 2023 г., 10:43:23

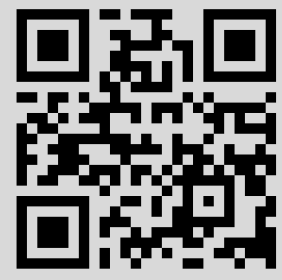




\title{
Сильная асимптотика нулей многочленов, ортогональных относительно комплексного веса
}

\author{
С. П. Суетин
}

1. Пусть $\rho$ - непрерывная комплекснозначная функция, заданная на отрезке $\Delta=$ $[-1,1]$. Если $\rho>0$ на $\Delta$ и удовлетворяет условию Дини-Липшица, то (см. [1], [2]) для многочленов $Q_{n}$, ортогональных на $\Delta$ с весом $\left(1-x^{2}\right)^{-1 / 2} \rho(x)$ и нормированных соответствующим образом, при $n \rightarrow \infty$ справедливы следующие асимптотические формулы Бернштейна:

$$
\begin{aligned}
& Q_{n}(z)=\Phi(z)^{n} D(z ; \rho)(1+o(1)), \quad z \in \overline{\mathbb{C}} \backslash \Delta, \\
& Q_{n}(x)=2(\rho(x))^{-1 / 2} \cos (n \arccos x+\theta(x ; \rho))+o(1), \quad x \in \Delta,
\end{aligned}
$$

где $D(z ; \rho)$ - функция Сегё, $\theta(x ; \rho)$ - “сдвиг фазы”, $\Phi(z)=z+\sqrt{z^{2}-1}-$ функция, обратная функции Жуковского $z=\frac{1}{2}(\zeta+1 / \zeta),|\zeta|>1, \Phi(\infty)=\infty, \zeta=\Phi(z)$. Соотношение (1) выполняется локально равномерно в $D=\overline{\mathbb{C}} \backslash \Delta$, соотношение (2) равномерно на $\Delta$. Случаю $\rho \equiv 1$ соответствуют классические полиномы Чебышёва $T_{n}(z)=\zeta^{n}+\zeta^{-n}$, нули которых вычисляются явно: $x_{k, n}=\cos \left(\frac{\pi}{2 n}+\frac{\pi k}{n}\right)$, $k=0, \ldots, n-1$. В частности, для нулей, ближайших к концам отрезка $\Delta$, имеем: $x_{0, n}=1-\pi^{2} /\left(4 n^{2}\right)+O\left(1 / n^{4}\right), x_{n-1, n}=-1+\pi^{2} /\left(4 n^{2}\right)+O\left(1 / n^{4}\right)$. Так как $\rho>0$, то $\operatorname{deg} Q_{n}=n$ и все $n$ нулей полинома $Q_{n}$ лежат на отрезке $\Delta$. Если функция $\rho$ голоморфна на $\Delta, \rho \in \mathscr{H}(\Delta)$, то (см. [3]-[5]) в (1), (2) величина $o(1)=o\left(\delta^{n}\right), \delta \in(0,1)$, и формула (2) позволяет приближенно с точностью $o\left(\delta^{n}\right)$ находить нули $Q_{n}$ как корни уравнения $\cos (n \arccos x+\theta(x ; \rho))=0$.

2. Изучение сходимости аппроксимаций Паде приводит к задаче об асимптотике многочленов, ортогональных относительно комплексного веса $\rho$ : такие многочлены естественным образом возникают как знаменатели диагональных аппроксимаций Паде [6; §2, п. 11], [7], [8]. Дж.Наттоллом [8], [3] было показано, что результаты С. Н. Бернштейна остаются в силе и для комплексного веса $\rho: \Delta \rightarrow \mathbb{C}^{*}=\mathbb{C} \backslash\{0\}$, $\rho \in \mathscr{H}(\Delta)$. Точнее, формула (1) остается неизменной, а вместо (2) имеем

$$
Q_{n}(x)=\Psi_{n}(x+i 0)+\Psi_{n}(x-i 0)+o\left(\delta^{n}\right), \quad x \in \Delta, \quad n \rightarrow \infty,
$$

где $\Psi_{n}(z):=\Phi(z)^{n} D(z ; \rho)$. Непосредственно из (1) вытекает, что $\operatorname{deg} Q_{n}=n$ при $n \geqslant n_{0}$, все нули $Q_{n}$ притягиваются к отрезку $\Delta$ и

$$
\frac{1}{n} \mu\left(Q_{n}\right) \rightarrow \frac{1}{\pi} \frac{d x}{\sqrt{1-x^{2}}}
$$

где $\mu\left(Q_{n}\right)$ - считающая мера для полинома $Q_{n}$. Однако для комплексного веса $\rho$ нули $Q_{n}$, вообще говоря, не лежат на $\Delta$. Поэтому формула (3) оказывается непригодной для приближенного решения уравнения $Q_{n}(z)=0$, в отличие от случая $\rho>0$. Тем самым, формулы типа (1)-(3) не позволяют проследить за асимптотическим поведением каждого из нулей полинома $Q_{n}$ при $n \rightarrow \infty$ и, в частности, не дают ответа на вопрос, с какой скоростью эти нули притягиваются к отрезку $\Delta$.

Основной результат настоящей работы - формула сильной асимптотики для $Q_{n}$ в "окрестности" отрезка $\Delta$. В частности, для веса $\rho(x)=e^{-i x}=\cos x-i \sin x$ из этой формулы вытекает индивидуальное описание асимптотического поведения каждого из $n$ нулей полинома $Q_{n}$ по отношению к нулям $x_{k, n}$ полинома Чебышёва. Иными словами, при $n \rightarrow \infty$ нули $Q_{n}$ фактически "нумеруются" нулями $T_{n}$.

Работа выполнена при поддержке РФФИ (грант № 05-01-01027) и гранта НШ-4466.2006.1. 
3. Пусть $\mathfrak{R}$ - риманова поверхность, заданная уравнением $w^{2}=z^{2}-1$ и двулистно накрывающая $\overline{\mathbb{C}} ; \mathbf{z}=(z, w)$ - точки на $\mathfrak{R}, \operatorname{pr}-$ каноническая проекция: $\operatorname{pr}(\mathbf{z})=z$. Отрезку $\Delta$ соответствует на $\mathfrak{R}$ замкнутый цикл $\boldsymbol{\Gamma}, \operatorname{pr}(\boldsymbol{\Gamma})=\Delta$. Функция $\Phi(z)$ и полином $Q_{n}(z)$ поднимаются на $\mathfrak{R}$ как функции от $\mathbf{z}$; так как $\rho \in \mathscr{H}(\Delta)$, то $\Psi_{n}$ голоморфна в некоторой окрестности $\boldsymbol{\Gamma}$.

Теорема 1. Если $\rho \in \mathscr{H}(\Delta), \rho(x) \neq 0$, то при некотором $\varepsilon>0$ равномерно по $\mathbf{z}$ таким, что $e^{-\varepsilon} \leqslant|\Phi(\mathbf{z})| \leqslant e^{\varepsilon}$, имеет место равенство

$$
Q_{n}(\mathbf{z})=\Psi_{n}(\mathbf{z})+\Psi_{n}\left(\mathbf{z}^{*}\right)+o\left(\delta^{n}\right), \quad n \rightarrow \infty,
$$

где $\mathbf{z}^{*}=\left(z, \mp \sqrt{z^{2}-1}\right)$ при $\mathbf{z}=\left(z, \pm \sqrt{z^{2}-1}\right)$, величина $\delta \in(0,1)$ и зависит от $\rho$.

Доказательство теоремы 1 проводится традиционным методом исследования асимптотических свойств ортогональных многочленов (см., например, [9], [5], [10]), основанным на изучении сингулярного интегрального уравнения Наттолла [3]. Очевидно, что соотношения (1)-(3) являются следствиями (4).

Из теоремы 1 вытекает

Teоpema 2. Ecлu $\rho(x)=e^{-i b x}, b>0, m o$ :

1) при $n \geqslant n_{0}$ все нули $z_{k, n}, k=0, \ldots, n-1$, многочлена $Q_{n}$ простые, лежат в верхней полуплоскости $\operatorname{Im} z>0$ и удовлетворяют неравенству $c_{1} / n^{2}<$ $g\left(z_{k, n}, \infty\right)<c_{2} / n$, где постоянные $c_{1}, c_{2}>0$ зависят от $b, g(z, \infty)$ - функиия Грина области $D$;

2) равномерно по $k=0, \ldots, n-1$ справедливо следующее асимптотическое представление:

$$
z_{k, n}=x_{k, n}+O\left(\frac{1}{n^{2}}\right)+i\left(\frac{b}{8 n} \sin \left(\frac{\pi}{2 n}+\frac{\pi k}{n}\right)+O\left(\frac{1}{n^{3}}\right)\right), \quad n \rightarrow \infty ;
$$

3) для нулей $Q_{n}$, ближайших к концам отрезка $\Delta$, имеем:

$$
z_{0, n}=x_{0, n}+i \frac{b \pi^{2}}{2 n^{3}}+O\left(\frac{1}{n^{4}}\right), \quad z_{n-1, n}=x_{n-1, n}+i \frac{b \pi^{2}}{2 n^{3}}+O\left(\frac{1}{n^{4}}\right) .
$$

Соотношение (5) означает, что образы $\zeta_{k, n}=\Phi\left(z_{k, n}\right)$ нулей $Q_{n}$ в плоскости переменного $\zeta$ асимптотически при $n \rightarrow \infty$ располагаются в корнях $2 n$-й степени из -1 .

Аналоги теорем 1 и 2 справедливы и для многочленов $Q_{n}(z ; f)$ - знаменателей диагональных аппроксимаций Паде функций вида $f=\hat{\rho}+r$, где $r \in \mathbb{C}(z) \cap \mathscr{H}(\Delta)$. Отметим, что первый результат о сильной асимптотике $m$ полюсов диагональных аппроксимаций Паде функции вида $f=\hat{\rho}+r$ в малой окрестности $m$-кратного полюса $f$ получен в [5; $\S 2$, п. 3, теорема 3$]:$ асимптотически эти полюсы располагаются в вершинах правильного т-угольника.

\section{Список литературы}

[1] С. Н. Бернштейн, О многочленах, ортогональных в конечном интервале, Гостехиздат Украины, Харьков, 1937. [2] Г. Сегё, Ортогональные многочлены, Физматгиз, М., 1962. [3] J. Nuttall, Constr. Approx., 6:2 (1990), 157-166. [4] A. I. Aptekarev, W. Van Assche, J. Approx. Theory, 129:2 (2004), 129-166. [5] А. А. Гончар, С. П. Суетин, Об аппроксимациях Паде мероморфных функиий марковского типа, Современные проблемы математики, $\mathbf{5}$, Матем. ин-т им. В. А. Стеклова РАН, М., 2004. [6] А. А. Гончар, Матем. сб., 97:4 (1975), 607-629. [7] Е. М. Никишин, В. Н. Сорокин, Рачиональные аппроксимачии и ортогональностъ, Наука, M., 1988. [8] J. Nuttall, J. Approx. Theory, 42:4 (1984), 299-386. [9] С. П. Суетин, УMH, 57:1 (2002), 45-142. [10] С. П. Суетин, Матем. сб., 198:6 (2007), 107-138.

С. П. Суетин (S. P. Suetin)

Математический институт им. В. А. Стеклова РАН

E-mail: suetin@mi.ras.ru
Представлено А. Г. Сергеевым Принято редколлегией 08.06.2007 